\title{
Síndrome de bridas amnióticas, a propósito de 3 casos clínicos
}

\author{
GLORIA DA SILVA ${ }^{1}$, FRANCISCO CAMMARATA-SCALISI ${ }^{2}$, \\ MERCEDES GONZÁLEZ-COIRA ${ }^{3}$, MARÍA ANGELINA LACRUZ, BELITZA RENDON ${ }^{5}$ \\ 1. Médico Genetista. Profesor Instructor de Genética Médica. Departamento de Puericultura y Pediatría. \\ Universidad de Los Andes. \\ 2. Profesor Instructor de Genética Médica. Departamento de Puericultura y Pediatría. Universidad de Los Andes. \\ 3. Biólogo Genetista. Profesor Asociado de Genética Médica. Departamento de Puericultura y Pediatría. \\ Universidad de Los Andes. \\ 4. Pediatra. Neurólogo Infantil. Profesor Asistente del Departamento de Puericultura y Pediatría. Universidad de Los Andes. \\ 5. Residente del Segundo año de Puericultura y Pediatría. Universidad de Los Andes.
}

\begin{abstract}
Amniotic band syndrome: 3 case-reports

Amniotic Band Syndrome is a sporadic condition with a spectrum of clinical presentations that include constriction rings, pseudosyndactily, amputations, multiple craniofacial - visceral - body wall defects and spontaneous abortion. The incidence ranges from 1: 1200 to 1: 15000 newborns, creating controversy regarding its pathogenesis. We report 3 cases with different clinical manifestations of this entity and review the different etiological hypotheses for this syndrome. Two main pathogenic mechanisms are proposed: the exogenous theory with early amnion rupture leading to fibrous bands that entrap the fetal body and the endogenous theory that establishes a germ plasm defect with vascular disruption and disturbance of morphogenesis during early gastrulation. However, the exact etiology of Amniotic Band Syndrome remains unknown and its natural evolution is unpredictable. The observed geographic difference in birth prevalence is useful in studying specific genetic and environmental factors involved. The management of this disease must be multidisciplinary and the outcome depends on malformations severity.

(Key words: Amniotic Band Syndrome, constriction rings, amputations, etiopathological, exogenous theory, endogenous theory)

Rev Chil Pediatr 2008; 79 (2): 172-180

\section{RESUMEN}

El síndrome de bridas amnióticas es una condición esporádica variable que presenta un espectro que incluye anillos de constricción, pseudosindactilia, amputaciones, múltiples alteraciones craneofaciales, viscerales, de la pared corporal y abortos espontáneos. El rango de incidencia es de 1 en 1200 a 1 en 15000
\end{abstract}

Trabajo recibido el 27 de noviembre de 2007, aceptado para publicación el 23 de febrero de 2008.

Correspondencia a:

Francisco Cammarata-Scalisi

E-mail: francocammarata@yahoo.it 
recién nacidos vivos. La patogenia del síndrome de bridas amnióticas es controversial. En el siguiente informe se reportan tres casos donde se describen las diferentes manifestaciones clínicas de esta entidad, además se revisaron las diferentes hipótesis etiológicas. Entre los dos mecanismos propuestos destacan la ruptura prematura del amnios (teoría exógena), que conlleva a la formación de bandas fibrosas que afectan el cuerpo fetal y la teoría endógena como defecto germinal, disrupción vascular y alteraciones en la morfogénesis durante la gastrulación temprana. Sin embargo, la etiología exacta del síndrome de bridas amnióticas es incierta y su curso natural es impredecible. Las diferencias geográficas observadas en las prevalencias de nacimiento puede ser una indicación útil para estudios de factores genéticos y ambientales candidatos. El manejo debe ser multidisciplinario y el pronóstico depende de la gravedad de las malformaciones.

(Palabras clave: Síndrome de bridas amnióticas, anillos de constricción, amputaciones, etiopatología, teoría exógena, teoría endógena).

Rev Chil Pediatr 2008; 79 (2): 172-180

\section{Introducción}

El síndrome de bridas amnióticas (SBA) abarca un amplio espectro de alteraciones congénitas que se caracterizan por anillos de constricción, pseudosindactilia, amputaciones y menos frecuentemente múltiples defectos craneofaciales, viscerales y alteraciones en la pared abdomino-toráxica ${ }^{1}$. Su incidencia varía entre 1 en 1200 a 1 en 15000 recién nacidos vivos ${ }^{1-4}$,y en abortos espontáneos puede llegar a ser de 178 en 10000 . Afecta ambos sexos por igual ${ }^{5}$, con una incidencia ligeramente elevada en descendientes afrocaribeños ${ }^{6}$. Su presentación tiene un carácter esporádico, aunque se han publicado algunos casos de recurrencia familiar ${ }^{4,7}$. El pronóstico depende de la severidad de las anormalidades y de la implicación de órganos comprometidos. La fisiopatología de este síndrome está en discusión, sin embargo, el estudio de los períodos críticos de la embriogénesis y organogénesis han proporcionado una explicación para entender los mecanismos que conducen a este síndrome ${ }^{8}$.

Una anomalía congénita en general puede ser debida a malformación, deformación y disrupción. El SBA se considera un error en la morfogénesis del tipo disrupción, que resulta de la destrucción secundaria o interferencia de un órgano o región corporal fetal que previamente tenía un desarrollo normal; pero se produce por algún factor extrínseco o por interferencias internas no heredables ${ }^{5}$. La causa más común de anomalías por disrupción es la ruptura prematura del amnios (RPA). La naturaleza y severidad de las consecuencias de esta ruptura se encuentra en relación con el tiempo de gestación. La presencia de bridas fibrosas de origen corioamniótico en la placenta es el elemento común que permite agrupar estas anomalías con el término de $\mathrm{SBA}^{2}$. En el siguiente reporte se hará referencia a tres casos clínicos en los cuales se describen diferentes manifestaciones del síndrome así como una revisión sobre las teorías etiopatológicas del mismo.

\section{Casos Clínicos}

\section{Caso 1}

Recién nacido masculino, producto de madre de 31 años de edad, primigesta, embarazo controlado, de término 38 semanas por fecha de última menstruación y ecografía, complicado con diagnóstico prenatal de hidrocefalia severa. Al momento del nacimiento presenta además múltiples malformaciones, por lo cual es trasladado a la Unidad de Alto Riesgo Neonatal.

Antecedentes familiares: Madre alfabeta, ocupación oficios del hogar, niega antecedentes clínicos y quirúrgicos de importancia. Padre de 26 años de edad, alfabeta, ocupación empleado en tienda textil, niega exposición a teratógenos ocupacionales. Sin antecedentes clínicos y quirúrgicos de importancia.

Antecedentes obstétricos: Niega ingesta 
de medicamentos durante la gestación. Se realizó ultrasonido de control prenatal donde se diagnosticó hidrocefalia severa a las 24 semanas de gestación. Por tal motivo, fue valorada por los servicios de Alto Riesgo Obstétrico, Neurocirugía y la Unidad de Genética Médica. Este último solicitó realización de ecografía morfogenética, el cual reportó embarazo con feto único de 30 semanas, y una hidrocefalia donde se evidenció aumento importante del diámetro biparietal; presentación cefálica, situación longitudinal, dorso anterior, lo que dificulta la visualización de estructuras faciales y miembro superior derecho.

Antecedentes perinatales: Producto de unión no consanguínea. Obtenido por cesárea

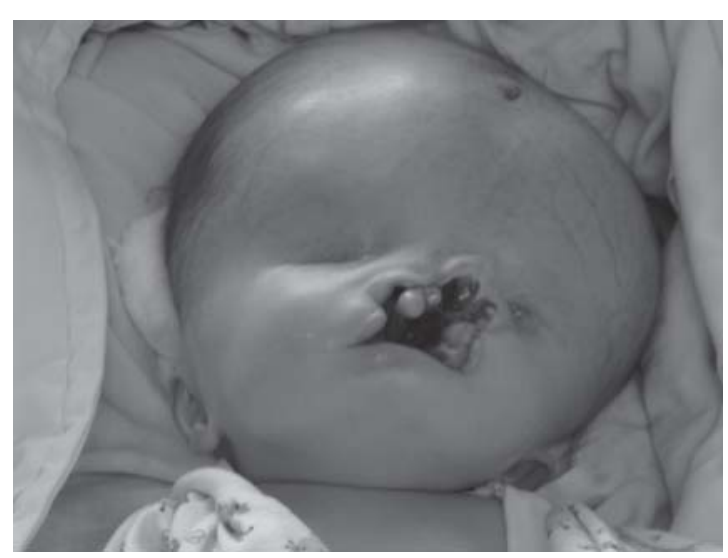

Figura 1. Caso 1. Recién nacido masculino con cráneo en forma de trébol, anoftalmía bilateral, malformación en línea media facial (Clasificación Tessier Número 3).

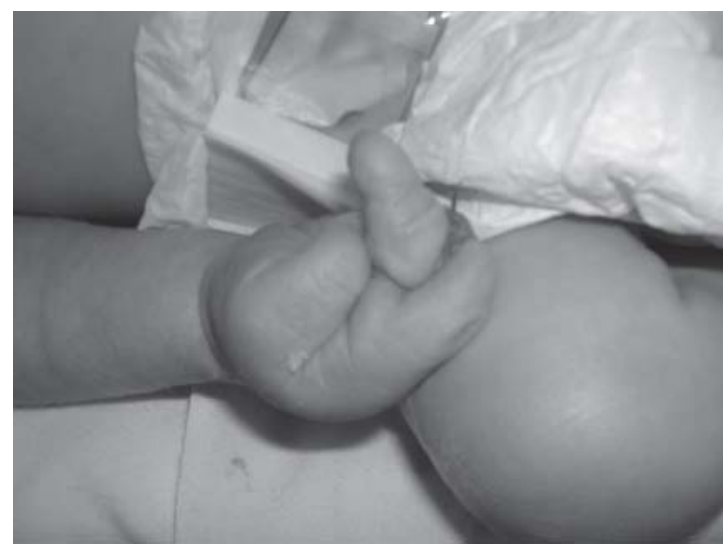

segmentaria bajo anestesia subaracnoidea por hidrocefalia severa; lloró y respiró espontáneamente al nacer, APGAR: 7-8 puntos, al primer y quinto minuto respectivamente, líquido amniótico claro con grumos, no presentó circular de cordón umbilical. Peso al nacer 4600 g y talla al nacer $52 \mathrm{~cm}$.

Examen físico: En su ingreso a la Unidad de Alto Riesgo Neonatal presentaba dificultad respiratoria; Ballard: 38 semanas. Al examen físico se observó notorio aumento de circunferencia cefálica, presentando perímetro de 47,5 cm, en relación al perímetro toráxico de $35 \mathrm{~cm}$; hidranencefalia, cráneo en forma de trébol, fontanelas tensas, con evidencia de dilataciones venosas y lesiones costrosas a nivel de región parieto-occipital, cuero cabelludo liso y escaso cabello, ausencia parcial de los huesos del cráneo (temporo-parietal izquierdo). Anoftalmía bilateral, hendiduras palpebrales pequeñas a predominio izquierdo, párpados ausentes. Malformación en línea media facial donde el defecto alcanza casi el ángulo interno de hendidura palpebral izquierda (Clasificación Tessier Número 3). Se apreciaba tabique nasal corto con tejido correspondiente a las fosas nasales, labio y paladar hendido bilateral, figura 1 . Pabellones auriculares bilaterales hipoplásicos de implantación baja. Extremidades: en mano derecha se apreciaba anillo de constricción en $3^{\circ}$ dedo, $2^{\circ}, 4^{\circ}$ y $5^{\circ}$ dedos hipoplásicos unidos por tejido filamentoso parduzco (pseudosindactilia), figuras $2 \mathrm{a}$ y $2 \mathrm{~b}$; en mano izquierda se evidencia disminución del tamaño de falange distal de $3^{\circ}$

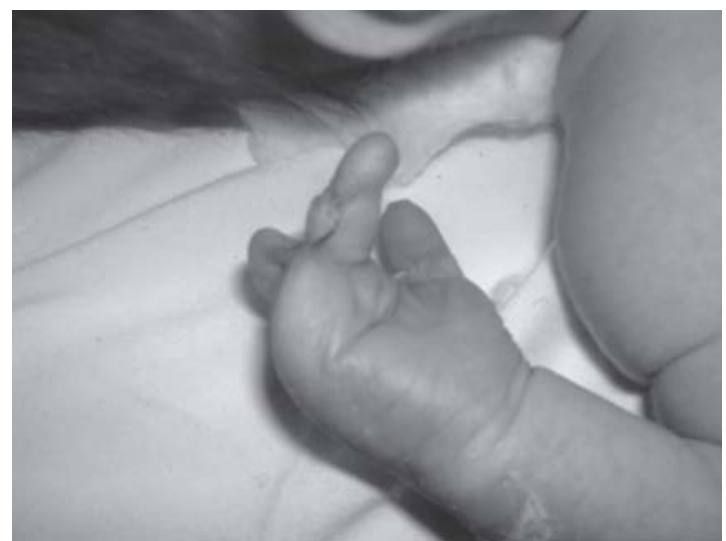

Figura 2a y 2b. Caso 1 . Mano derecha se aprecia anillo de constricción en $3^{\circ}$ dedo, pseudosindactilia en $2^{\circ}, 4^{\circ}$ y $5^{\circ}$. 
dedo. A nivel de miembros inferiores: pie equino varo bilateral, a predominio izquierdo, se observa anillo de constricción en tercio distal de pierna, así como en región proximal de cada dedo y ausencia de segundo ortejo.

Exámenes de laboratorio: Hematología completa dentro de los límites normales. Química sanguínea: hiperglicemia, creatinina normal. Resultados de hemocultivo y cultivo de LCR: negativo.

Tomografía Axial Computarizada de cráneo evidenció hidranecefalia. Ultrasonido abdominorenal no presentó alteraciones y el estudio ecocardiográfico reportó foramen oval permeable y aneurisma del tabique interauricular.

Presentó evolución no favorable, falleciendo al mes y once días de edad.

\section{Caso 2}

Lactante menor masculino, producto de madre de 38 años de edad, segunda gesta, embarazo controlado, de término (41 semanas más dos días por fecha de última menstruación), complicado con proceso alérgico en piel y escabiosis, recibió tratamiento tópico con crotamitón y calamina; además de tratamiento multivitamínico por vía oral durante la gestación. Referido del Servicio de Ortopedia y Traumatología para su evaluación en la consulta de Genética Médica por presentar amputaciones desde el $2^{\circ}$ al $5^{\circ}$ dedo en mano izquierda.

Antecedentes familiares: Madre alfabeta, ocupación enfermera, niega antecedentes clínicos y quirúrgicos de importancia. Padre de 43 años de edad, alfabeta, ocupación pintor automotriz, refiere exposición a teratógenos ocupacionales (plaguicidas), sin embargo, el estudio toxicológico no reportó alteraciones; niega antecedentes quirúrgicos.

Antecedentes perinatales: Producto de unión no consanguínea. Obtenido por cesárea segmentaria bajo anestesia subaracnoidea por útero cicatrizal, la causa de la cesárea anterior fue desproporción céfalo-pélvica. Peso al nacer: 3300 g y talla al nacer: 50 cm. Respiración espontánea al momento del nacimiento, niega cianosis e ictericia durante período neonatal.

Examen físico: Realizado a los 30 días de nacido. Peso y talla acorde para la edad. Normocéfalo, aumento de volumen en región temporo-

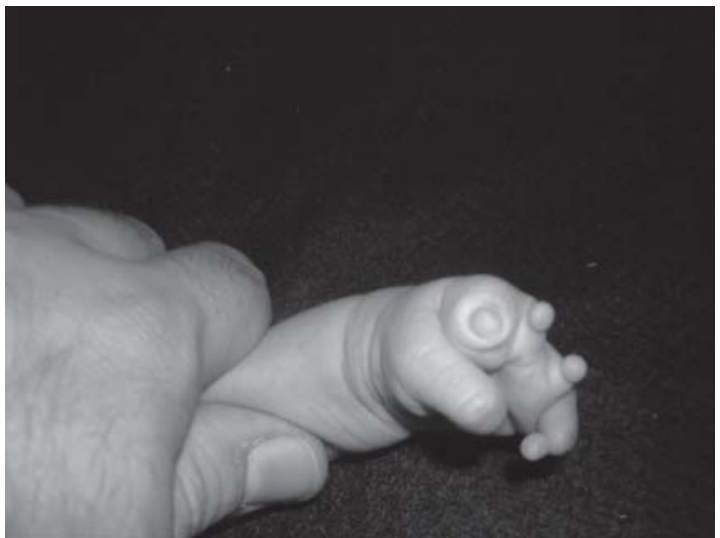

Figura 3. Caso 2. Mano izquierda: se evidencia amputaciones del $2^{\circ}$ al $5^{\circ}$ dedo con anillos de constricción.

occipital izquierda, nariz con puente cóncavo y antevertida; cavidad oral: paladar ojival. Pabellones auriculares con adecuada implantación y rotados hacia atrás. Tórax simétrico, auscultación cardiopulmonar normal. Abdomen: diastasis de los rectos. Extremidades: mano izquierda: $1^{\circ}$ dedo sin alteraciones, se evidencia amputaciones del $2^{\circ}$ al $5^{\circ}$ dedo con anillos de constricción, figura 3. El resto de las de las extremidades sin evidencia de alteración. Neurológico conservado.

Exámenes realizados: Estudio radiológico de mano izquierda: $1^{\circ}$ dedo sin alteración y sólo se evidenció falange proximal de $2^{\circ}$ dedo. Ultrasonido transfontanelar reportó ventrículo lateral izquierdo dilatado; no se observaron imágenes sugestivas de hemorragia. Resonancia Magnética Nuclear Cerebral evidenció hipoagenesia del cuerpo calloso con dilatación ventricular izquierda. Ultrasonido abdomino-renal reportó leve pielocalectasia renal izquierda. Ecocardiograma: corazón estructural y funcionalmente normal.

\section{Caso 3}

Recién nacido masculino, producto de madre de 16 años de edad, primigesta, embarazo controlado, de término: 41 semanas por fecha de última menstruación; complicado con cefalea y edema en miembros inferiores en el último trimestre, no precisa aumento de cifra en tensión arterial; además refiere exposición a Carbamatos en aerosol durante la gestación. Referi- 


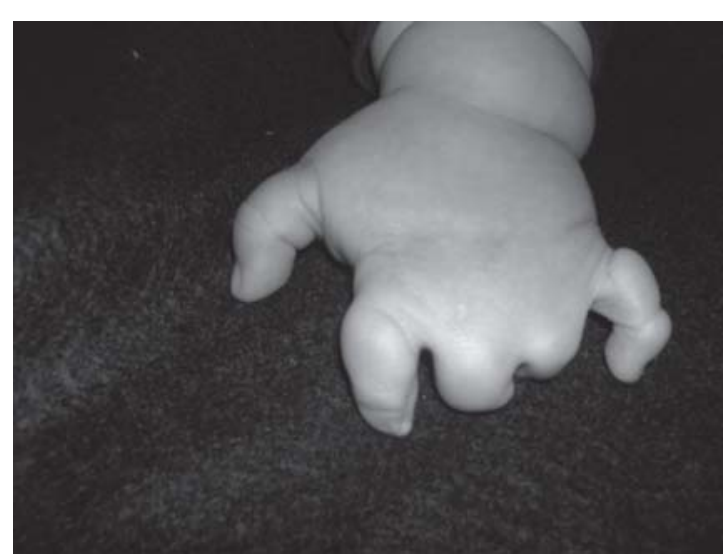

Figura 4a. Caso 3. Mano izquierda: se aprecia uña rudimentaria en $2^{\circ}$ dedo, amputación distal en $3^{\circ}$ y $4^{\circ}$ dedo y anillo de constricción en $5^{\circ}$ dedo, así como en antebrazo.

do de la Unidad de Bajo Riesgo Neonatal para su evaluación en la consulta de Genética Médica por presentar alteraciones estructurales en mano izquierda y ambos pies.

Antecedentes familiares: Madre alfabeta, ocupación estudiante, refiere gastritis, niega antecedentes quirúrgicos. Padre de 25 años de edad, alfabeta, ocupación comerciante, niega antecedentes clínicos y quirúrgicos de importancia, así como exposición a teratógenos ocupacionales.

Antecedentes perinatales: Producto de unión no consanguínea. Obtenido por cesárea segmentaria bajo anestesia subaracnoidea por distocia de dilatación durante el trabajo de parto. Peso al nacer: $3300 \mathrm{~g}$ y talla al nacer: $47 \mathrm{~cm}$. Respiración espontánea al momento del nacimiento, niega cianosis e ictericia hasta el momento de la consulta.

Examen físico: Realizado al quinto día de nacido. Normocéfalo, hipertricosis en región frontotemporal bilateral, cejas escasas; nariz antevertida; boca: paladar ojival. Pabellones auriculares de implantación normal, rotados hacia atrás; cuello corto. Tórax simétrico, auscultación cardiopulmonar normal. Abdomen: hernia umbilical. Extremidades: en mano izquierda: se apreciaba uña rudimentaria en $2^{\circ}$ dedo, amputación distal en $3^{\circ}$ y $4^{\circ}$ dedo y anillos de constricción en $5^{\circ}$ dedo y antebrazo ipsilateral, figura 4a. Pie derecho: $1^{\circ}$ dedo hipoplásico, anillo de constricción en $4^{\circ}$ dedo; pie izquierdo:

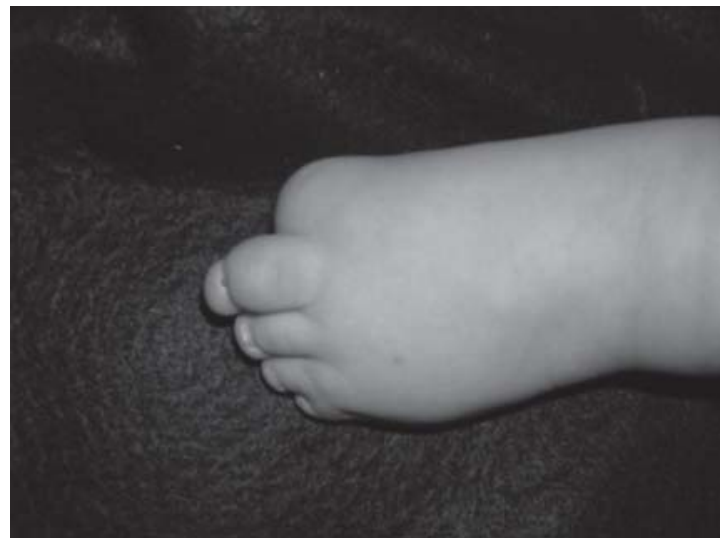

Figura 4b. Pie izquierdo: amputación de $1^{\circ}$ dedo, anillo de constricción en $2^{\circ}$ dedo y sindactilia en $4^{\circ}$ y $5^{\circ}$ dedo.

amputación de $1^{\circ}$ dedo, anillo de constricción en $2^{\circ}$ dedo y sindactilia en $4^{\circ}$ y $5^{\circ}$ dedo, figura 4b. Neurológico conservado. El paciente fue revalorado a los dos meses de edad presentando adecuado desarrollo pondo-estatural y psicomotor.

Exámenes realizados: Informe radiológico de mano izquierda y ambos pies reportó adecuada formación de metacarpianos, falange proximal y distal $1^{\circ}$ dedo, y falanges proximales del $2^{\circ}$ al $5^{\circ}$ dedo. Ausencia de falanges distales del $2^{\circ}$ al $4^{\circ}$ dedo, con alteración en la morfología de las falanges medias de los mismos. Pie derecho: falanges proximales del $2^{\circ}$ al $5^{\circ}$, dedo con distorsión de la morfología de la falange proximal del $1^{\circ}$ dedo y ausencia distal del mismo; pie izquierdo: ausencia de falange distal de $1^{\circ}$ dedo. Ultrasonido transfontanelar no evidenció alteración para el momento de realizar el estudio. Ultrasonido renal reportó leve pielocalectasia renal izquierda.

\section{Discusión}

Algunas de las hipótesis explicativas del SBA fueron propuestas por Torpin ${ }^{9}$, en el cual la RPA es un evento precipitante ${ }^{6}$. El amnios es la membrana que cubre la cara fetal de la placenta formando la superficie externa del cordón umbilical, surge como una capa de células epiteliales planas sobre una delgada capa de 
mesoblasto. El embrión y el amnios están rodeados por la membrana coriónica que desarrolla vellosidades en la segunda semana posterior a la fertilización y recibe la vascularización de los vasos de la alantoides para formar la placenta. Con el crecimiento embrionario, el amnios se distiende con el fluido amniótico y se fusiona con el mesoblasto coriónico. La RPA provoca variados efectos según la etapa de gestación fetal. Cuando es perforado precozmente, el defecto se expande de manera rápida pudiendo llegar hasta la zona de inserción placentaria del cordón umbilical. La ruptura del amnios permite el pasaje de líquido a la interfase amniocoriónica y el embrión es expuesto a la superficie interna de la cavidad coriónica estimulando la proliferación de bandas mesenquimatosas adherentes. Estas bandas amnióticas pueden adherirse a la superficie gelatinosa del embrión, limitando sus movimientos, como así también atrapar y estrangular las estructuras anatómicas fetales, provocando compresión seguida de isquemia y necrosis. El resultado sería una amputación si la estructura fetal se encontrara en desarrollo, mientras que, si estuviese ya formada, daría lugar a un anillo de constricción, esto sucedería a partir de la séptima semana de gestación ${ }^{2}$. Esta teoría también conocida como exógena ha sido acreditada por observación ecográfica de bridas en embarazos que culminaron con la presencia de alteraciones en niños compatibles con el síndrome. El análisis de placentas con signos de RPA tanto de recién nacidos con este síndrome como de abortos espontáneos, apoyan también esta teoría ${ }^{10}$.

No obstante, esta teoría es refutada con base sustentada en evidencias clínicas y experimentales que indican que hay una elevada prevalencia de anormalidades viscerales internas, difíciles de explicar por la secuencia de bandas propuesta por Torpin ${ }^{5}$. Además se han presentado otras malformaciones que presentan un revestimiento amniótico histológicamente normal e indemne, y el frecuente hallazgo de defectos fetales por disrupción que no están en contacto con bridas. Los experimentos con sustancias vasoactivas en el modelo animal, han reproducido estas características avalando histológicamente, que las hemorragias preceden a la constricción de los miembros, amputaciones entre otros ${ }^{5,11}$. Por esto se piensa que la patogenia involucra la lesión del mesénquima, células endoteliales de los vasos superficiales del embrión, del amnios y la ruptura de células epiblásticas; por lo que la formación de bandas amnióticas sería un fenómeno tardío y secundario $^{5}$.

Otra hipótesis propuesta por Torpin, fue la denominada "displasia focal fetal”, o teoría endógena, la cual señala que los defectos asociados con el SBA obedecen a errores focales de desarrollo en la formación del tejido conectivo de los miembros ${ }^{5}$. Esta no gozó de aceptación porque no podía explicar la gran variedad de lesiones, asimetría, además los defectos estaban presentes en estructuras que se formaban en muy diferentes momentos y que éstos a su vez, eran derivados de las diferentes capas germinales ${ }^{6}$.

La teoría de Streeter, reexaminada por Bamforth, la cual no está en contraposición con la primera teoría de Torpin, ya mencionada, considera que la fuerza mecánica de las bandas amnióticas no sólo comprometería la vascularización fetal sino que también interferiría con el desarrollo del disco germinal. Ésta explicaría casos con anormalidades craneanas por interferencia en el cierre del neuroporo anterior o migración de los tejidos de la cresta neural cefálica y algunos otros con anomalías internas, como cardiopatías o defectos del cierre de las paredes corporales ${ }^{2,12,13}$.

La RPA se ha relacionado con ciertos factores como: traumatismos abdominales maternos, intervenciones quirúrgicas antes o durante la gestación, uso de dispositivo intrauterino ${ }^{5}$, malformaciones uterinas, enfermedades del colágeno: (osteogénesis imperfecta y síndrome de Ehlers-Danlos), ingestión de fármacos:

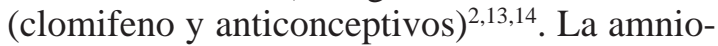
centesis, especialmente en casos de biopsias de vellosidades coriónicas, también ha sido involucrada5,13,15,16; sin embargo, en la mayoría de los casos, el SBA es esporádico, con muy bajo riesgo de recurrencia y sin causa demostrable $^{12}$, como los casos presentados en este reporte.

Una evaluación fetopatológica de 18 casos con SBA, por Moerman y $\mathrm{col}^{7}$, discernieron 
tres tipos de lesiones: bandas constrictivas de tejido, adhesiones amnióticas y patrones más complejos de la anomalía designados como complejo de pared cuerpo-miembro. Las bandas constrictivas son causadas por la RPA, donde se compromete subsecuentemente las partes fetales y sobre todo las extremidades. Las adhesiones son en particular el resultado de una amplia fusión entre las partes fetales interrumpidas, sobre todo las cefálicas y una membrana amniótica intacta. La mayoría de los defectos craneofaciales (encefaloceles y hendiduras faciales) que ocurren en estos fetos no son causados por las bandas amnióticas constrictivas sino el resultado de una secuencia de disrupción vascular con o sin la adhesión cefalo-amniótica. Estas observaciones confirman el hecho de que la ruptura del amnios no es una condición indispensable para el desarrollo del complejo de pared cuerpo-miembro. Sin embargo, este complejo es con frecuencia complicado por la ruptura del amnios sin soporte con la formación de bandas constrictivas. El concepto que conside- ra las tres lesiones en cuestión, como una simple entidad patogenética es errónea y conducirá inevitablemente a una discusión interminable entre los seguidores de las dos teorías que prevalecen. Las teorías de Streeter y Torpin no son mutuamente exclusivas, pero explican la etipatogenia de los diversos tipos de lesiones.

El diagnóstico neonatal del SBA es realizado sólo en 29 al 50\% de los casos. El hallazgo clínico más común en este síndrome consiste en anillos de constricción de los dedos de las manos y los pies en 77\%, los cuales pueden presentar múltiples anomalías asociadas ${ }^{5}$. Las malformaciones craneofaciales pueden ser bizarras y las alteraciones en órganos internos son infrecuentes ${ }^{6}$. En la tabla 1 se muestran los posibles hallazgos clínicos presentes en el SBA y su presencia en los pacientes evaluados. Entre las alteraciones genéticas a las que se debe hacer diagnóstico diferencial con SBA tenemos: el síndrome del “bebé Michelín” o síndrome de los surcos cutáneos circunferenciales múltiples benignos, síndrome de Adams-Oliver

Tabla 1. Hallazgos clínicos presentes en los pacientes evaluados con SBA

\begin{tabular}{|c|c|c|c|}
\hline Área afectada/Tipo de defecto & Caso 1 & Caso 2 & Caso3 \\
\hline \multicolumn{4}{|l|}{ Cráneo-facial } \\
\hline Defecto de cierre de tubo neural: anencefalia & - & - & - \\
\hline Acalvaria & + & - & - \\
\hline Encefalocele & - & - & - \\
\hline Hidranecefalia & + & - & - \\
\hline Hidrocefalia & - & - & - \\
\hline Dilatación ventricular (no específica) & - & + & - \\
\hline Estenosis del acueducto de Silvio & - & - & - \\
\hline Craneosinostosis (Cráneo en forma de trébol) & + & - & - \\
\hline Anoftalmía & + & - & - \\
\hline Hendidura facial & - & - & - \\
\hline Labio/Paladar hendido & + & - & - \\
\hline \multicolumn{4}{|l|}{ Alteración en la pared abdomino-toráxica } \\
\hline Ectopia cordis & - & - & - \\
\hline Alteración cardíaca & + & - & NP \\
\hline Onfalocele & - & - & - \\
\hline \multicolumn{4}{|l|}{ Extremidades superiores } \\
\hline Anillos de constricción & + & + & + \\
\hline Sindactilia & - & - & - \\
\hline Pseudosindactilia & + & - & - \\
\hline Amputación & + & + & + \\
\hline \multicolumn{4}{|l|}{ Extremidades inferiores } \\
\hline Anillos de constricción & + & - & + \\
\hline Sindactilia & - & - & + \\
\hline Peudosindactilia & - & - & - \\
\hline Amputación & + & - & + \\
\hline
\end{tabular}

NP: No precisado 
y el espectro de síndromes de hipogenesia oromandibular-miembros ${ }^{2}$. El estudio ultrasonográfico prenatal permite hacer el diagnóstico al visualizar las bandas amnióticas en un feto que muestra limitaciones en su movimiento y la presencia de deformidades ${ }^{5,17}$. El diagnóstico se puede confirmar por la ruptura crónica del corion en cortes histológicos placentarios. El diagnóstico diferencial debe hacerse con respecto a cicatrices o sinequias intrauterinas secundarias a intervenciones en la cavidad uterina, el síndrome del cordón umbilical corto, y tabique mulleriano, entre otros ${ }^{18,19}$.

La secuencia de ADAM (deformidad amniótica, adhesión y mutilación) es una condición heterogénea, con un amplio espectro de anomalías, en la cual están igualmente implicadas causas intrínsecas como defectos germinales, disrupción vascular y alteraciones en la morfogénesis, alternado con causas extrínsecas como la ruptura de bandas amnióticas. Orioli y col ${ }^{20}$, identificaron 270 casos en Brasil, con alguna evidencia en piel de constricción y además presentaban defectos sugestivos de secuencia de ADAM. Este estudio se realizó entre 3020896 nacidos vivos y muertos, que abarca el período 1982-1998. En la mitad de los casos presentados, hubo mutilación (reducción) y deformidad (anillo de constricción) afectando la porción distal de los dedos, sin la presencia de otros defectos asociados. Acrania, encefalocele, hendiduras faciales, coloboma del párpado, y celosomía estaban significantemente asociados a las lesiones en piel. La incidencia fue de uno por cada 11200 nacimientos, manteniendo una tendencia estable durante el período de realización del estudio. Un número importante de casos se presentó en personas que residían en áreas geográficas con altitud importante, madres con una historia prenatal de enfermedad febril aguda, uso de medicación y sangrado vaginal durante el primer trimestre del embarazo. Se encontró además una alta frecuencia en niños primogénitos, prematuros, de bajo peso al nacer para la edad gestacional y presentación no cefálica. La diferencia geográfica observada podía ser una indicación útil para estudiar factores genéticos y ambientales específicos como candidatos a la susceptibilidad a la secuencia ADAM.
El SBA exhibe una diversa variedad de manifestaciones clínicas que deben ser conocidas para realizar el correcto diagnóstico clínico durante el período neonatal y a su vez descartar la coexistencia de otras alteraciones que pueden estar presentes. En los casos presentados en este informe, como otros publicados en la literatura predominan las lesiones a nivel de extremidades: anillos de constricción y amputaciones, a pesar de esto la evaluación clínica y paraclínica debe ser completa y de forma sistematizada. Los pacientes evaluados fueron referidos a la Unidad de Genética Médica de la Universidad de Los Andes, en un período de un año, lo que representa una frecuencia de 1 por 6000 nacimientos. El lugar de nacimiento, así como de residencia de los progenitores y abuelos de los propósitos es diferente, no son cercanos y de distintas características geográficas.

El ser producto primogénito fue el único factor de riesgo que estuvo presente en dos de los casos, tabla 2, lo que coincide con otros estudios donde el SBA, no se pueden establecer las posibles causas de la entidad. En los casos comentados en este apartado debemos resaltar la exposición a Carbamatos durante la gestación en la madre del caso 3 y la exposición a plaguicidas en el padre del caso 2 , a pesar que el estudio toxicológico no reportó anormalidad. Las edades maternas fueron diversas, desde una adolescente de 16 años, hasta una gestante tardía de 38 años, casos 3 y 2 respectivamente, la paciente del caso 1 presentaba para el momento de la concepción 31 años. Esta última sólo presentó como factor de riesgo ser primigesta, sin embargo, el producto presentó las mayores alteraciones anatómicas de los casos expuestos, situación que corrobora lo difícil de establecer las posibles causas etiológicas y a su vez la poca relación entre estas y la presentación clínica. Los diversos factores que pueden influir en la aparición de este esporádico síndrome deben ser aún ampliamente estudiados para establecer su importancia, donde la obtención de los antecedentes es un punto importante para su búsqueda. Además en este reporte se estudiaron las diversas teorías etiopatogénicas propuestas por Torpin y Streeter, donde ambas explican los diferentes tipos de lesiones que se pueden presentar en el SBA. 
Tabla 2. Factores de riesgo que pueden influir en la aparición de SBA en los pacientes estudiados

\begin{tabular}{|c|c|c|c|c|}
\hline \multicolumn{2}{|c|}{ Factor posible de riesgo } & Caso 1 & Caso 2 & Caso 3 \\
\hline \multicolumn{2}{|c|}{ Intervención quirúrgica abdominal en la madre } & - & - & - \\
\hline \multicolumn{2}{|c|}{ Traumatismo abdominal materno } & - & - & - \\
\hline \multicolumn{2}{|c|}{ Dispositivo intrauterino } & - & - & - \\
\hline \multicolumn{2}{|c|}{ Malformaciones uterinas } & - & - & - \\
\hline \multirow{2}{*}{\multicolumn{2}{|c|}{$\begin{array}{l}\text { Amniocentesis } \\
\text { Enfermedad del colágeno: }\end{array}$}} & - & - & - \\
\hline & & & & \\
\hline \multicolumn{2}{|c|}{ Osteogénesis imperfecta } & - & - & - \\
\hline & Síndrome de Ehlers-Danlos & - & - & - \\
\hline \multirow[t]{2}{*}{ Fármacos: } & Clomifeno & - & - & - \\
\hline & Anticonceptivos orales & - & - & - \\
\hline \multicolumn{2}{|c|}{ Zona de residencia rural } & - & - & + \\
\hline \multicolumn{2}{|c|}{ Zona de residencia con altitud considerable } & - & + & - \\
\hline \multicolumn{2}{|c|}{$(>1500$ m.s.n.m) } & $(5-40)$ & (1 708) & $(160)$ \\
\hline \multicolumn{2}{|c|}{ Enfermedad febril durante el embarazo } & - & - & - \\
\hline \multicolumn{2}{|c|}{ Producto primogénito } & - & - & + \\
\hline \multicolumn{2}{|c|}{ Bajo peso al nacer } & - & - & - \\
\hline \multicolumn{2}{|c|}{ Presentación no cefálica } & - & - & - \\
\hline
\end{tabular}

m.s.n.m.: metros sobre el nivel del mar

\section{Referencias}

1.- Pedersen TK, Thomsen SG: Spontaneous resolution of amniotic bands. Ultrasound Obstet Gynecol 2001; 18: $673-4$.

2.- Bibas $H$, Atar M, Espíndola M: Síndrome de bridas amnióticas. Arch Argent Pediatr 2002; 100: 240-4.

3.- Ronderos-Dumit D, Briceño F, Navarro H, Sánchez N: Endoscopic release of limb constriction rings in utero. Fetal Diagn Ther 2006; 21: 255-8.

4.- Martín D: Caracterización clínica de la secuencia de bridas amnióticas. Rev Cubana de Genética Humana 1999; 1: 2-5.

5.- Rivas-López R, Juárez-Azpilcueta A, Islas L, Durán MA, Oviedo I: Síndrome de bandas amnióticas asociado a secuencia Potter. Un caso de autopsia. Rev Mex Pediatr 2005; 72; 78-81.

6.- Chandran S, Lim MK, Yu VY: Fetal acalvaria with amniotic band syndrome. Arch Dis Child Fetal Neonatal 2000; 82: 11-3.

7.- Moerman P, Fryns JP: Constrictive amniotic bands, amniotic adhesions and limb-body wall complex: discrete disruption sequences with pathogenetic overlap. Am J Med Genet 1992; 42: 470-9.

8.- Bodamer OA, Popek EJ, Bacino C: Atypical presentation of amniotic band sequence. Am J Med Genet 2001; 100: 100-2.

9.- Torpin R: Amniochorionic mesoblastic fibrous strings and amnionic bands: associated constricting fetal malformations or fetal death. Am J Obstet Gynecol 1965; 91: 65-75.

10.- Van Allen MI, Siegel-Bartelt J, Dixon J, Zuker RM, Clarke HM, Toi A: Constriction bands and limb reduction defects in two newborns with fetal ultrasound evidence for vascular disruption. Am J Med Genet 1992; 44: 598-604

11.- Herva R: Amniotic adhesion malformation syndrome: fetal and placental pathology. Theratology 1984; 29 : 11-6.

12.- Levy PA, Adam HM: Amniotic bands. Pediatr Rev 1998; 19: 249-51.

13.- Bamforth JS: Amniotic band sequence: Streeter's hypothesis reexamined. Am J Med Genet 1992; 44: 280-7.

14.- De la Cabada C, Naranjo H, Duque L: Bandas constrictivas prenatales, presentación de un caso y revision de la literatura. Síndrome de las bandas amnióticas. Derm Ven 1992; 30: 121-5.

15.- Kohn G: The amniotic band syndrome: A possible complication of amniocentesis. Prenat Diagn 1987; 8: 303-7.

16.- Lage JM: Questionable role of amniocentesis in the etiology of amniotic band formation: A case report. J Reprod Med 1988; 33: 71-4.

17.- Randel SB: Amniotic Sheets. Radiology 1988; 166: 633-6.

18.- Van Allen MI: Limb-body wall complex: II. Limb and spine defects. Am J Med Genet 1987; 38: 549-53.

19.- Pons A, Sáez R, Sepúlveda W: Brida amniótica, sinequia intrauterina y tabique mulleriano: Etiopatogenia, diagnóstico diferencial y pronóstico. Rev Chil Ultrasonog 2005; 8: 51-8.

20.- Orioli IM, Ribeiro MG, Castilla EE: Clinical and epidemiological studies of amniotic deformity, adhesion, and mutilation (ADAM) sequence in a South American (ECLAMC) population. Am J Med Genet A 2003; 118: 135-45. 